\title{
Can the frontal tibiofemoral alignment be assessed on anteroposterior knee radiographs?
}

\author{
M. Sgroi ${ }^{1}$ M. Faschingbauer ${ }^{1} \cdot$ H. Reichel $^{1} \cdot$ T. Kappe $^{1}$
}

Received: 7 May 2015/ Accepted: 31 March 2016/Published online: 16 April 2016

(c) The Author(s) 2016. This article is published with open access at Springerlink.com

\begin{abstract}
Background The aim of total knee arthroplasty is, amongst others, the reconstruction of a physiological axis of the leg with a tibiofemoral angle in the frontal plane of an average of $6^{\circ}$. The aim of this study is to clarify how much of the bone length on the femur and tibia has to be reproduced on anteroposterior (AP) knee radiographs in order to determine the leg's alignment after a total knee arthroplasty.

Materials and methods We analyzed the postoperative hip-to-ankle (HTA) radiographs of 100 patients who had undergone a total knee arthroplasty at our institution.

Results There were strong correlations between the measured values on HTA and $20 \mathrm{~cm}$ bone length [lateral distal femur angle (LDFA) $r=0.887$, medial proximal tibial angle (MPTA) $r=0.874$, tibiofemoral angle (TFA) $r=0.888]$, but not between the measurements on HTA and $10 \mathrm{~cm}$ (LDFA $r=0.267$, MPTA $r=0.102$, TFA $r=0.161)$. There were significant differences between all measurements both on HTA and $20 \mathrm{~cm}$ and on HTA and $10 \mathrm{~cm}$, with the exception of the LDFA between HTA and $10 \mathrm{~cm}(p=0.085)$ and of the MPTA between HTA and $20 \mathrm{~cm}(p=0.227)$. The intra- and inter-observer correlations were both high.
\end{abstract}

The study was approved by our institutional review board.

M. Sgroi

sgroi.mirco@yahoo.de

1 Department for Orthopaedic Surgery, University of Ulm, Oberer Eselsberg 45, 89081 Ulm, Germany
Conclusion If preoperatively crude axis deviations are excluded, the tibiofemoral angle on AP knee radiographs can be determined with an accuracy of $\pm 2.6^{\circ}$ if at least $20 \mathrm{~cm}$ length of bone is reproduced (measured from the femoral and tibial joint line). Due to the high $95 \%$ confidence intervals and bearing in mind that deviations greater than $3^{\circ}$ may lead to inferior clinical results, however, it appears inappropriate to determine lower limb alignment with anteroposterior radiographs.

Level of evidence Level 2.

Keywords Knee arthroplasty · Alignment - Full strut . AP knee recording

\section{Introduction}

The traditional aim of total knee arthroplasty (TKA) is to restore a neutral axis of the leg with a tibiofemoral angle (TFA) of $5^{\circ}-7^{\circ}$ [14]. Malalignment after TKA, especially in reference to the tibial component, has been shown to lead to negative biomechanical as well as clinical consequences, e.g., early loosening [11]. The TFA is traditionally measured on hip-to-ankle (HTA) radiographs. This is used both for preoperative surgical planning and to verify the correct reconstruction of the postoperative axis. The HTA has been shown to be prone to errors in rotation and location of the central beam as well as insufficient weight application [8]. Even though recent studies confirm that it is not possible to derive the TFA from short knee radiographs [4], it is still unclear how much of the femur and the tibia has to be depicted in order to measure the TFA accurately. The aim of this study, therefore, was to determine what bone length of the femur and tibia has to be exposed in order to determine the TFA correctly. 


\section{Materials and methods}

Between August 2010 and December 2011, 428 consecutive patients with degenerative osteoarthritis of the knee underwent primary TKA at our department. HTA radiographs were done preoperatively in all patients. Patients meeting the following criteria were excluded from the study: (1) a history of fracture of the lower limb; (2) a history of lower limb axis correction surgery; (3) crude axis deviation (excessive femur varum or curvature of the tibia); and (4) for technical reasons (e.g., malrotated X-ray). After exclusion of these criteria, 100 HTA radiographs were selected at random and included in the present study (57 women and 43 men). All the HTA radiographs investigated were performed between days 3 and 7 after surgery. The average age of the patients was 68.6 years (range 49-86 years).

All the recordings analyzed were HTA radiographs obtained on graduated-grid $30 \times 90 \mathrm{~cm}$ cassettes. The recordings were performed in standing position and in full extension at a distance of 3 meters (Fig. 1). Reference bodies of $2.5 \mathrm{~cm}$ diameter were routinely attached to the patient's skin at the level of the knee. Rotation was controlled by determining the amount of the superposition of fibular head and lateral tibial plateau. Radiographs were deemed acceptable if $1 / 3$ of the fibular head was superimposed. Malrotated radiographs were excluded from the study.

First, femoral component orientation (lateral distal femur angle, LDFA) was determined by measuring the angle between a line connecting the most distal points on the surfaces of the femoral condyles and a line connecting the centre of the femoral canal at two points $30 \mathrm{~cm}$ apart, according to Paley [12]. Tibial component orientation (medial proximal tibial angle, MPTA) was then measured as the angle between a line connecting the most proximal medial and proximal lateral points of the tibial component to a line connecting the centre of the tibial medullary canal at two points $30 \mathrm{~cm}$ apart. The TFA was determined as the angle between the two intramedullary axes described above. HTA radiographs were then imaginarily cut at intervals of 20 and $10 \mathrm{~cm}$ length of bone, measured from the joint line, on both the femoral and the tibial sides. The same measurements as above were then carried out after every imaginary shortening and the above parameters measured again (Fig. 2).

In order to allow determination of the intra-observer and inter-observer reliability, 20 HTA radiographs were examined twice by two orthopaedic surgeons. The survey was carried out using the AGFA planning system (Agfa HealthCare GmbH, Bonn, Germany).

The collected data were analyzed using Pearson's correlation coefficient (PCC) using simple linear regression,

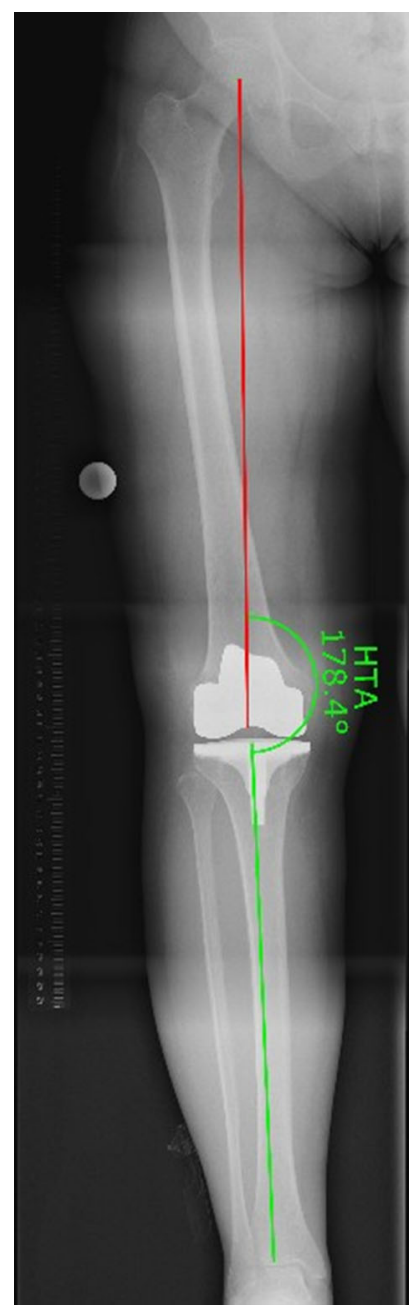

Fig. 1 Measurement of HTA angle (HTA angle between the mechanical axis of the femur and mechanical axis of the tibia)

the $t$ test and $95 \%$ confidence intervals. The differences were considered significant if the $p$ value was less than 0.05. The statistical analysis was performed using SSPS.

\section{Results}

The results of the measurements are presented in Table 1 . There was a strong correlation between the measured values on HTA and $20 \mathrm{~cm}$ bone length (LDFA $r=0.887$, $p<0.001$; MPTA $r=0.874, p<0.001$; TFA $r=0.888$, $p<0.001$ ), but not between the measurements on HTA and $10 \mathrm{~cm}$ (LDFA $r=0.267 p=0.007$, MPTA $r=0.102$ $p=0.314$, TFA $r=0.161 p=0.109)$. There were significant differences between all measurements on HTA and $20 \mathrm{~cm}$ and on HTA and $10 \mathrm{~cm}$, with the exception of the LDFA between HTA and $10 \mathrm{~cm}(p=0.085)$ and the MPTA between HTA and $20 \mathrm{~cm}(p=0.223)$. The $95 \%$ 
Fig. 2 Measurement of TFA, MPTA and LDFA on a HTA radiograph, $\mathbf{b} 20 \mathrm{~cm}$ bone length radiograph, $\mathbf{c} 10 \mathrm{~cm}$ bone length radiograph. TFA tibial femoral angle, $L D F A$ lateral distal femur angle, MPTA medial proximal tibial angle, HTA hip-to-ankle radiograph
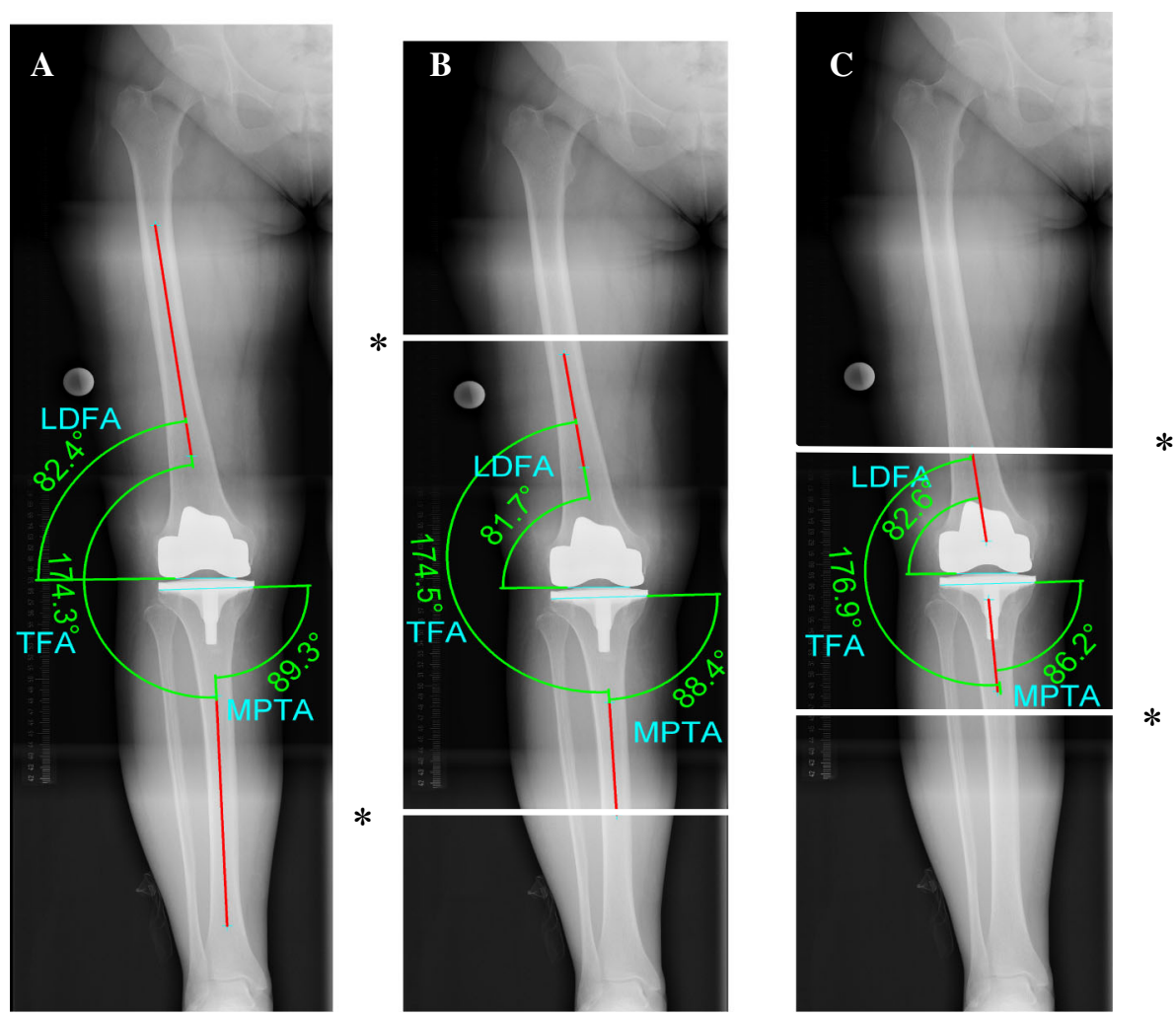

Table 1 Measurement of TFA, MPTA and LDFA on HTA and on both short anteroposterior knee radiographs

\begin{tabular}{lrcc}
\hline & \multicolumn{1}{l}{ HTA } & 20 cm bone length & $10 \mathrm{~cm}$ bone length \\
\hline TFA & $173.23 \pm 2.86$ & $172.91 \pm 2.60$ & $176.09 \pm 3.29$ \\
LDFA & $83.88 \pm 2.19$ & $83.61 \pm 1.84$ & $84.37 \pm 2.39$ \\
MPTA & $90.75 \pm 1.74$ & $90.85 \pm 1.51$ & $88.38 \pm 2.19$ \\
\hline
\end{tabular}

TFA tibial femoral angle, LDFA lateral distal femur angle, $M P T A$ medial proximal tibial angle, HTA hip-to-ankle radiograph

Table 2 Inter-observer reliability for TFA, MPTA and LDFA on HTA and on both short anteroposterior knee radiographs

\begin{tabular}{llll}
\hline & HTA & $20 \mathrm{~cm}$ bone length & $10 \mathrm{~cm}$ bone length \\
\hline TFA & $0.926^{*}$ & $0.882^{*}$ & $0.729^{* *}$ \\
LDFA & $0.918^{*}$ & $0.842^{*}$ & $0.844^{*}$ \\
MPTA & $0.892^{*}$ & $0.841^{*}$ & $0.892^{*}$ \\
\hline
\end{tabular}

TFA tibial femoral angle, LDFA lateral distal femur angle, MPTA medial proximal tibial angle, HTA hip-to-ankle radiograph

$* p>0.001, * * p>0.003$

confidence interval for the LDFA was $2.0^{\circ}$ (HTA and $20 \mathrm{~cm}$ ) and $5.5^{\circ}$ (HTA and $10 \mathrm{~cm}$ ), for the MPTA $1.7^{\circ}$ (HTA and $20 \mathrm{~cm}$ ) and $5.1^{\circ}$ (HTA and $10 \mathrm{~cm}$ ) and for the TFA $2.6^{\circ}$ (HTA and $20 \mathrm{~cm}$ ) and $7.8^{\circ}$ (HTA and $10 \mathrm{~cm}$ ). The inter-observer correlation was high (Table 2).

\section{Discussion}

The aim of the current study was to determine the length of femur and tibia which has to be reproduced on anteroposterior radiographs in order to accurately measure lower limb alignment. The results suggest that exposing $20 \mathrm{~cm}$ of the femur and tibia will introduce a rather negligible measurement error of -2.6 to $2.6^{\circ}$.

Determining alignment before and after TKA is essential for surgical planning, execution of the operation and postoperative evaluation of the treatment result [2]. Different studies have shown that a postoperative malalignment contributes to reducing the longevity of TKA [3].

Both short anteroposterior knee radiographs and HTA radiographs are used to check lower limb alignment after TKA. Short knee radiographs do not reveal extra-articular deformity or deviations in femoral neck length, resulting in $6^{\circ} \pm 1^{\circ}$ angle deviation. Therefore, only the anatomical, not the mechanical axis can be derived from short knee radiographs. In a study by McGrory et al. [10] it was shown that the mechanical axis plays a rather subordinate role in comparison to the TFA in axis reconstruction.

The concept of neutral alignment after TKA has recently been challenged, however. In a study by Matziolis et al. [9] it was postulated that a varus malalignment after TKA has no effect on clinical outcome. In another study by Bellemans [1] it was proposed to re-establish the patient's own 
native baseline type of alignment. In comparison to the predominance of data supporting a neutral mechanical axis and approximately $5^{\circ}-7^{\circ}$ valgus anatomic alignment, there is little support for choosing any other aim [7].

There is a paucity of data on whether the TFA can be determined with sufficient accuracy on postoperative anteroposterior knee radiographs. In 1988, Petersen et al. compared the TFA measured on anteroposterior knee radiographs with measurements on HTA radiographs in 50 patients after total knee arthroplasty and found a discrepancy of $1.4^{\circ}$ with a standard deviation (SD) of $2.2^{\circ}$ [13]. In a similar study on 83 patients, Skyttä et al. [15] measured a difference of $1.4^{\circ}$ with an SD of $1.4^{\circ}$ for the TFA. The two studies have shown that both TFA measurement techniques correlate strongly with each other.

Hirschmann et al. [5] measured the intra- and inter-observer reliability of measurements of the position of the components after total knee replacement using plane radiographs and axial two-dimensional and three-dimensional reconstructed computed tomography (CT) images. They found that three-dimensional reconstructed images are sufficiently reliable to measure the position and orientation of the components. The derivation of postoperative alignment after TKA using $\mathrm{CT}$ is associated with a higher radiation exposure for the patient, and for this reason this method cannot be considered a standard procedure.

The present study has several limitations. First, outliers were preliminarily prevented by excluding patients with gross alignment deviation. This appeared intuitive to us as we felt this would increase the clinical applicability of the data. Even if HTA radiographs were not acquired on a routine basis, they would at least be obtained in patients posing challenges for intraoperative limb alignment correction. Second, we artificially cut HTA films to short radiographs in order to prevent having our results affected by additional deviations in projection and rotation. In clinical practice, the differences between shorter and HTA radiographs may therefore be even higher than the results of the present study suggests. We were interested in the magnitude of the effect of limiting exposure on measurement accuracy, and artificially cutting HTA films appeared to perfectly exclude further influencing factors while limiting unnecessary X-ray exposure to patients.

In summary, this study shows that it is theoretically possible to derive lower limb alignment from shorter knee radiographs if at least $20 \mathrm{~cm}$ of the tibia and femur are depicted and if gross extra-articular deformity has previously been excluded. However, this study also suggests that a measurement error of $-2.6^{\circ}$ to $2.6^{\circ}$ already arises by the exposure of $20 \mathrm{~cm}$ of the femur and the tibia. Due to these high $95 \%$ confidence intervals and bearing in mind that deviations greater than $3^{\circ}$ may lead to inferior clinical results [6], however, it appears inappropriate to determine lower limb alignment with shorter knee radiographs. Therefore, we do not recommend determining lower limb alignment with short anteroposterior radiographs; HTA radiographs should be considered the gold standard for routine practice.

\section{Compliance with ethical standards}

Conflict of interest Each author certifies that he or she, or a member of their immediate family, has no commercial associations (e.g., consultancies, stock ownership, equity interest, patent/licensing arrangements, etc.) that might pose a conflict of interest in connection with the submitted article.

Ethical standards Obtaining informed consent from the patients involved was waived by the Research Ethics Committee. All procedures involving human participants were in accordance with the 1964 Helsinki declaration and its later amendments. The study was approved by the Research Ethics Committee (registration number: 174/12).

Open Access This article is distributed under the terms of the Creative Commons Attribution 4.0 International License (http://creati vecommons.org/licenses/by/4.0/), which permits unrestricted use, distribution, and reproduction in any medium, provided you give appropriate credit to the original author(s) and the source, provide a link to the Creative Commons license, and indicate if changes were made.

\section{References}

1. Bellemans J, Colyn W, Vandenneucker H, Victor J (2012) The Chitranjan Ranawat award: is neutral mechanical alignment normal for all patients? The concept of constitutional varus. Clin Orthop Relat Res 470(1):45-53

2. Benjamin $\mathbf{J}$ (2006) Component alignment in total knee arthroplasty. Instr Course Lect 55:405-412

3. Berend ME, Ritter MA, Meding JB, Faris PM, Keating EM, Redelman $\mathrm{R}$ et al (2004) Tibial component failure mechanisms in total knee arthroplasty. Clin Orthop Relat Res 428:26-34

4. Felson DT, Cooke TD, Niu J, Goggins J, Choi J, Yu J et al (2009) Can anatomic alignment measured from a knee radiograph substitute for mechanical alignment from full limb films? Osteoarthritis Cartilage 17(11):1448-1452

5. Hirschmann MT, Konala P, Amsler F, Iranpour F, Friederich NF, Cobb JP (2011) The position and orientation of total knee replacement components: a comparison of conventional radiographs, transverse 2D-CT slices and 3D-CT reconstruction. J Bone Joint Surg Br 93(5):629-633

6. Jeffery RS, Morris RW, Denham RA (1991) Coronal alignment after total knee replacement. J Bone Joint Surg Br 73(5):709-714

7. Lombardi AV Jr, Berend KR, Ng VY (2011) Neutral mechanical alignment: a requirement for successful TKA: affirms. Orthopedics 34(9):e504-e506

8. Lonner JH, Laird MT, Stuchin SA (1996) Effect of rotation and knee flexion on radiographic alignment in total knee arthroplasties. Clin Orthop Relat Res 331:102-106

9. Matziolis G, Adam J, Perka C (2010) Varus malalignment has no influence on clinical outcome in midterm follow-up after total knee replacement. Arch Orthop Trauma Surg 130(12):1487-1491

10. McGrory JE, Trousdale RT, Pagnano MW, Nigbur M (2002) Preoperative hip to ankle radiographs in total knee arthroplasty. Clin Orthop Relat Res 404:196-202 
11. Miehlke R, Schwenen M (1980) Roentgenological assessment of knee joint arthroplasties (author's transl)]. Z Orthop Ihre Grenzgeb 118(1):66-72

12. Paley D, Pfeil J (2000) Principles of deformity correction around the knee. Der Orthopade 29(1):18-38

13. Petersen TL, Engh GA (1988) Radiographic assessment of knee alignment after total knee arthroplasty. J Arthroplasty 3(1):67-72
14. Ritter MA, Faris PM, Keating EM, Meding JB (1994) Postoperative alignment of total knee replacement. Its effect on survival. Clin Orthop Relat Res 299:153-156

15. Skytta ET, Lohman M, Tallroth K, Remes V (2009) Comparison of standard anteroposterior knee and hip-to-ankle radiographs in determining the lower limb and implant alignment after total knee arthroplasty. Scand J Surg 98(4):250-253 